\title{
Global Trend of Breast Cancer Mortality Rate: A 25-Year Study
}

\author{
Nasrindokht Azamjah ${ }^{1}$, Yasaman Soltan-Zadeh ${ }^{2 *}$, Farid Zayeri ${ }^{3}$
}

\begin{abstract}
Background: breast cancer is the most common cause of cancer death for women worldwide. In the past two decades, published epidemiological reports in different parts of the world show significant increase in breast cancer mortality rate. The aim of this study was to determine the 25 -year trend of breast cancer mortality rate in 7 super regions defined by the Health Metrics and Evaluation (IHME), i.e. Sub-Saharan Africa, North Africa and Middle East, South Asia, Southeast Asia and East Asia and Oceania, Latin America and Caribbean, Central Europe and Eastern Europe and Central Asia, High-income. Methods: Our study population consisted of 195 world countries in the IHME pre-defined seven super regions. The age-standardized mortality rates from 1990 to 2015 were extracted from the IHME site. The reference life table for calculating mortality rates was constructed based on the lowest estimated age-specific mortality rates from all locations with populations over 5 million in the 2015 iteration of GBD. To determine the trend of breast cancer mortality rate, a generalized linear mixed model was fitted separately for each IHME region and super region. Results: Statistical analysis showed a significant increase for breast cancer mortality rate in all super regions, except for High-income super region. For total world countries, the mean breast cancer mortality rate was 13.77 per 100,000 in 1990 and the overall slope of mortality rate was 0.7 per 100,000 from 1990 to 2015. The results showed that Latin America and Caribbean the highest increasing trend of breast cancer mortality rate during the years 1990 to 2015 (1.48 per 100,000). Conclusion: In general, our finding showed a significant increase in breast cancer mortality rate in the world during the past 25 years, which could be due to increase in incidence and prevalence of this cancer. Low this increasing trend is an alarm for health policy makers in all countries, especially in developing countries and low-income regions which experienced sharp slopes of breast cancer mortality rate.
\end{abstract}

Keywords: Breast cancer- mortality rate- Trend analysis

Asian Pac J Cancer Prev, 20 (7), 2015-2020

\section{Introduction}

Cancer has one of the highest mortality rates worldwide. In 2015, there were estimated 17.5 million cancer cases followed by 8.7 million deaths (Global Burden of Disease Cancer Collaboration, 2017). Among different kinds of cancers, breast cancer is one the most important health problems around the world (Sharma, 2019).

One of the most comprehensive assessment in 2004 showed that breast cancer was the most common cancer worldwide among women. It was the leading cause of death for women in the world, the second in Africa and Southeast Asia after Cervical cancer while it was in the fifth place in West Pacific (World Health Organization, 2008). The more recent studies confirm that these statistics are still remaining true. In 2012, breast cancer was the most common cancer type with $13.8 \%$ of all cancer cases (Ferlay et al., 2013). A study in China, India and Russia, showed that breast cancer was the second cause of death among women after Lung cancer in 2014 (Goss et al., 2014).

Because of several contributing factors, there has been a general increase in breast cancer cases in recent years. A study in 2017 showed an increase of cases by $33 \%$ from 2005 to 2015 , while population growth was the reason for $12.6 \%$, aging population for $16.4 \%$ and $4.1 \%$ was because of age-specific cases (Global Burden of Disease Cancer Collaboration 2017). In 2012 in the European Union, the number of death cases decreased by 91,000 (Ferlay et al., 2013), however from 2005 to 2015, there was an increase of $21.3 \%$ in breast cancer mortality from 439,800 to 533,600 (Wang et al., 2016).

Regarding the increasing trend of breast cancer around the world, we decided to conduct the present study in order to assess the trend of this cancer in nearly all world countries. In this context, the present study aims to present a longitudinal analysis of breast cancer mortality rate in seven super regions of the world.

${ }^{1}$ Department of Administrative Science, Faculty of Shariaty, Tehran Branch, Technical and Vocational University (TVU), ${ }^{3}$ Proteomics Research Center and Department of Biostatistics, Faculty of paramedical Sciences, Shahid Beheshti University of Medical Sciences, Tehran, Iran, ${ }^{2}$ Department of Information Studies, University College London, Gower Street, London, WC1E 6BT, UK. *For Correspondence: y.soltan-zadeh@ucl.ac.uk 


\section{Materials and Methods}

Database used in the study

In this study, the data on age standardized breast cancer mortality rate from the GBD database according to a list of countries in world was used. The information includes age standardized mortality rate due to breast cancer in 195 countries, from 1990 to 2015 (in 5-year intervals; i.e. years 1990, 1995, 2000, 2005, 2010, 2015). The reference life table for calculating mortality rates was constructed based on the lowest estimated age-specific mortality rates from all locations with populations over 5 million in the 2015 iteration of GBD. We initially considered seven areas which were classified by the GBD: Sub-Saharan Africa, North Africa and Middle East, South Asia, Southeast Asia and East Asia and Oceania, Latin America and Caribbean, Central Europe and Eastern Europe and Central Asia, High-income. GBD has also classified countries under different regions and super regions. The mortality rate (per 100,000) due to breast cancer for each super regions, were considered as the main outcome of the study (Institute for Health Metrics and Evaluation, 2018).

\section{Statistical methods}

For descriptive purposes, the breast cancer mortality rate were presented using the mean (SD), separately for different region (Table 1) and super regions (Table 2).

To evaluate the trend of breast cancer mortality rate around the world, a general linear mixed effects model was fitted for each GBD super region. The model can be written as:

$$
E\left(y_{i t}\right)=\beta_{0}+\beta_{1} \text { time }+b_{0 i}
$$

Where $y_{i t}$ indicates the breast cancer mortality rate for ith country in th year under study. In additional, $b_{0 i}$ shows the random intercept of the model which distributed as: $N\left(0 ; \sigma_{b}^{2}\right)$.The model parameters, $\beta_{0}$ and $\beta_{1}$, are common fixed effect coefficients for model intercept and slope.

\section{Results}

Table 1 and table 2 show the descriptive statistics for mortality rate, respectively for the IHME regions and super regions during 1990 to 2015.

Comparing the average for regions reveals that Western Europe (year 1995) and Western Sub-Saharan Africa (year 1990) regions had, respectively, the highest and lowest average $(38.04,5.70)$.

Regarding table 2, the High-income super region had the highest and South Asia super region had the lowest breast cancer mortality rate during 25 -year period, under study (32.56 vs 6.12)

In the next step, we fitted the described random effects model in methods section to the data, separately for each super region. Table 3 displays the obtained results.

The obtained results in Table 3 show that the world countries experienced a mean breast cancer mortality rate of 13.77 per 100,000 in year 1990. In addition, these countries had an annual mean increase of 0.7 per 100,000 from year 1990 to 2015 .

Comparing the estimated intercepts reveals that the High-income and South Asia countries had, respectively, the highest and lowest breast cancer mortality rate in year 1990 (32.24 and 5.58 per 100,000). Moreover, the estimated slopes show that Latin America and Caribbean the highest increasing trend of breast cancer mortality rate during the years 1990 to 2015 (1.48 per 100,000). According the obtained p-value (last column table 3), one can conclude that all the super regions had significant positive trend of breast cancer mortality rate $(p<0.01)$, except for the High- income super region which had a non-significant negative slope during the study period $(\mathrm{p}=0.489)$.

Figure 1 displays the growth trajectories for seven super regions (each line shows mortality rate trend over

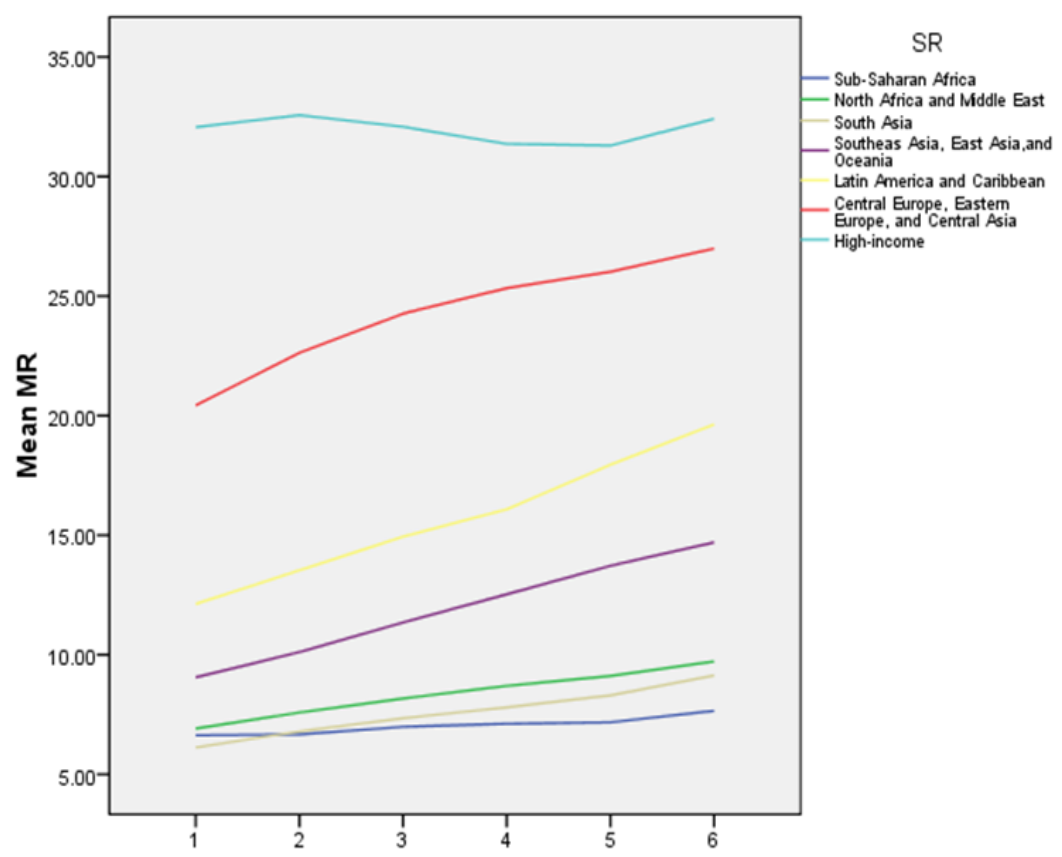

Figure 1. Breast Cancer Mortality Rate Trajectories of 195 World Countries 
Table 1. Mean (SD) for Breast Cancer Mortality Rate for Each IHME Region from 1995 to 2015

\begin{tabular}{lcccccc}
\hline & & & & \\
& & & \\
Region & 1990 & 1995 & 2000 & 2005 & 2010 & 2015 \\
\hline Eastern Sub-Saharan Africa & $5.94(1.75)$ & $6.13(1.78)$ & $6.19(1.62)$ & $5.80(1.49)$ & $6.00(1.62)$ & $6.65(1.81)$ \\
Western Sub-Saharan Africa & $5.70(1.24)$ & $5.75(1.24)$ & $5.85(1.32)$ & $5.85(1.50)$ & $5.98(1.66)$ & $6.34(1.81)$ \\
Southern Sub-Saharan Africa & $7.63(3.62)$ & $7.58(3.66)$ & $9.64(4.34)$ & $11.56(4.64)$ & $10.95(4.44)$ & $11.35(5.45)$ \\
Central Sub-Saharan Africa & $10.30(2.79)$ & $10.01(2.86)$ & $9.92(3.21)$ & $9.93(3.13)$ & $10.14(2.60)$ & $10.67(2.63)$ \\
North Africa and Middle East & $6.91(3.15)$ & $7.58(3.43)$ & $8.17(3.67)$ & $8.70(3.99)$ & $9.12(4.06)$ & $9.72(4.00)$ \\
South Asia & $6.12(3.49)$ & $6.79(4.29)$ & $7.35(4.81)$ & $7.80(5.17)$ & $8.30(5.20)$ & $9.14(5.25)$ \\
Southeast Asia & $7.73(1.21)$ & $8.75(1.63)$ & $10.05(1.82)$ & $11.33(2.11)$ & $12.19(2.56)$ & $12.70(2.91)$ \\
East Asia & $8.06(4.09)$ & $9.22(4.70)$ & $10.71(5.57)$ & $11.99(6.15)$ & $13.22(6.66)$ & $14.30(7.35)$ \\
Oceania & $10.46(5.02)$ & $11.42(5.57)$ & $12.38(5.98)$ & $13.40(5.85)$ & $14.65(5.94)$ & $15.64(6.33)$ \\
Central Latin America & $6.17(2.13)$ & $6.92(2.31)$ & $7.56(2.47)$ & $8.41(2.74)$ & $9.52(3.19)$ & $10.73(3.58)$ \\
Andean Latin America & $5.97(0.96)$ & $6.59(0.87)$ & $7.03(0.42)$ & $7.45(0.34)$ & $8.20(0.63)$ & $8.89(0.88)$ \\
Caribbean & $16.52(6.62)$ & $18.44(7.43)$ & $20.41(8.28)$ & $21.76(8.72)$ & $24.23(9.69)$ & $26.36(10.59)$ \\
Tropical Latin America & $8.58(1.41)$ & $9.55(1.73)$ & $10.81(1.45)$ & $12.43(0.79)$ & $13.89(0.65)$ & $15.33(1.03)$ \\
Eastern Europe & $24.69(3.46)$ & $28.46(4.21)$ & $29.50(5.03)$ & $30.29(4.70)$ & $30.48(4.01)$ & $31.92(4.18)$ \\
Central Europe & $24.74(9.11)$ & $27.04(9.55)$ & $29.25(9.94)$ & $30.70(9.32)$ & $31.69(8.41)$ & $32.53(8.78)$ \\
Central Asia & $10.88(7.08)$ & $11.71(7.69)$ & $13.01(9.20)$ & $13.71(9.74)$ & $14.36(10.10)$ & $15.15(10.69)$ \\
Southern Latin America & $25.54(13.08)$ & $26.61(13.61)$ & $27.85(13.84)$ & $28.54(13.43)$ & $30.00(13.22)$ & $32.33(13.77)$ \\
Western Europe & $37.57(10.00)$ & $38.04(9.08)$ & $37.06(8.15)$ & $35.77(6.96)$ & $35.20(7.14)$ & $36.00(7.59)$ \\
High-income Asia Pacific & $9.48(3.23)$ & $10.57(3.93)$ & $11.93(4.48)$ & $13.51(4.56)$ & $15.28(4.96)$ & $17.04(5.06)$ \\
Australasia & $31.67(4.56)$ & $31.32(3.48)$ & $30.27(3.64)$ & $28.97(2.97)$ & $29.04(3.11)$ & $30.11(3.49)$ \\
High-income North America & $28.52(8.85)$ & $28.50(8.17)$ & $27.83(6.16)$ & $27.27(5.92)$ & $26.80(6.14)$ & $28.24(6.86)$ \\
\hline & & & & & &
\end{tabular}

time for each super region). Except High-income (which had a non-significant negative slope during the study period), these trajectories show similar increasing trends for these super regions. "Sub-Saharan Africa" shows a mild decrease from 1990 to 1995, while during 1995 to 2010 here is an insignificant yet steady increase, with a considerable increase for 2015. "North Africa and Middle East" region and "South Asia" region have steady increasing rate from 1990 to 2015. This steady increasing rate is much sharper for "Southeast Asia, East Asia, and Oceania" region, "Latin America and Caribbean" region and "Central Europe, Eastern Europe, and Central Asia" region. For "High-income" region is mildly unsteady, after an increase in 1995, there is decrease to 2010, while there is a relatively sharp increase for 2015 .

\section{Discussion}

Breast cancer is an important health concern with an increasing trend of prevalence and mortality rate globally. In the present study, the data obtained from GLMM showed a positive slope of 0.7 for the total of countries of the world which means that the breast cancer mortality rate increases (on average) about seven in million every 5 years in the world.

Regarding Table 1, one can conclude that during 1990 to 2015 the highest increasing rate belongs to the Caribbean region, with 16.52 in 1990 to 26.36 in 2015. The decreasing rate most belongs to the Western Europe with 37.57 in 1990 to 36.00 in 2015 .

This study, (Table 2), indicates that the highest increasing rate belongs to the Latin American and Caribbean super region (from 12.12 to 19.64) while the lowest increasing rate belongs to High-income super

Table 2. Mean (SD) for Breast Cancer Mortality Rate for Each IHME Super Region from 1995 to 2015

\begin{tabular}{|c|c|c|c|c|c|c|}
\hline \multirow[b]{2}{*}{ Super region } & \multicolumn{5}{|c|}{ Year } & \multirow[b]{2}{*}{2015} \\
\hline & 1990 & 1995 & 2000 & 2005 & 2010 & \\
\hline Sub-Saharan Africa & $6.63(2.52)$ & $6.67(2.46)$ & $6.99(2.76)$ & $7.11(3.16)$ & $7.18(3.00)$ & $7.66(3.24)$ \\
\hline North Africa and Middle East & $6.91(3.15)$ & $7.58(3.43)$ & $8.17(3.67)$ & $8.70(3.99)$ & $9.12(4.06)$ & $9.72(4.00)$ \\
\hline South Asia & $6.12(3.49)$ & $6.79(4.29)$ & $7.35(4.81)$ & $7.80(5.17)$ & $8.30(5.20)$ & $9.14(5.25)$ \\
\hline Southeast Asia, East Asia, and Oceania & $9.06(4.40)$ & $10.11(4.90)$ & $11.35(5.43)$ & $12.53(5.63)$ & $13.72(5.95)$ & $14.70(6.47)$ \\
\hline Latin America and Caribbean & $12.12(7.16)$ & $13.53(8.01)$ & $14.94(8.92)$ & $16.08(9.36)$ & $17.95(10.39)$ & $19.64(11.25)$ \\
\hline $\begin{array}{l}\text { Central Europe, Eastern Europe, and } \\
\text { Central Asia }\end{array}$ & $20.43(9.74)$ & $22.63(10.76)$ & $24.27(11.45)$ & $25.33(11.49)$ & $26.02(11.25)$ & $26.99(11.61)$ \\
\hline High-income & $32.06(12.94)$ & $32.56(12.37)$ & $32.08(11.30)$ & $31.36(10.01)$ & $31.29(9.56)$ & $32.42(9.64)$ \\
\hline
\end{tabular}


Table 3. Breast Cancer Mortality Rate (Per 100,000) and Estimates from the GLMMs by Global Burden of Disease Study Super Regions

\begin{tabular}{llccc}
\hline Super regions & Parameter & Estimate & SE & P \\
\hline Sub-Saharan Africa & Intercept & 6.36 & 0.42 & $<0.001$ \\
& Slope & 0.19 & 0.03 & $<0.001$ \\
North Africa and Middle East & Intercept & 6.45 & 0.79 & $<0.001$ \\
& Slope & 0.55 & 0.04 & $<0.001$ \\
South Asia & Intercept & 5.58 & 1.95 & 0.004 \\
& Slope & 0.57 & 0.15 & $<0.001$ \\
Southeast Asia, East Asia, and Oceania & Intercept & 7.89 & 1.01 & $<0.001$ \\
& Slope & 1.15 & 0.07 & $<0.001$ \\
Latin America and Caribbean & Intercept & 10.52 & 1.61 & $<0.001$ \\
& Slope & 1.48 & 0.09 & $<0.001$ \\
Central Europe, Eastern Europe, and Central Asia & Intercept & 19.87 & 2.02 & $<0.001$ \\
& Slope & 1.26 & 0.09 & $<0.001$ \\
High-income & Intercept & 32.24 & 1.86 & $<0.001$ \\
& Slope & -0.078 & 0.11 & 0.489 \\
Total & Intercept & 13.77 & 0.86 & $<0.001$ \\
& Slope & 0.7 & 0.04 & $<0.001$ \\
\hline
\end{tabular}

region (from 32.06 to 32.42 ). A less than 3 in 100,000 increase could be observed in the North Africa and Middle East region.

Based on the American Cancer Society reports, breast cancer has the second highest cancer death after lung cancer for women. One out of eight American women may experience this cancer which is an average risk of $12 \%$. In 2017 , it was estimated that 252,710 invasive and 63,410 non-invasive (CIS) cases would be diagnosed, while it was predicted about 40,610 deaths due to this cancer. From 1989 to 2015, there was a 39\% drop in mortality rate. Since 2007 the mortality rate amongst women over 50 declined while it has stayed steadily for those under 50 (American Cancer Society, 2017).

Cancer Research UK shows that in 2014 there were 357,000 new cancer cases, with 181,000 males and 176,000 females. Breast cancer was the most common cancer for women in the UK. In compare to early 1990s there was an increase rate of $12 \%$ for all cancers, with the increase rate of $16 \%$ for females and $4 \%$ for males (Cancer Research UK, 2014b). The trend for breast cancer was 55,200 new cases which was $15 \%$ of all new cases, of which 390 cases were males and 54,800 females. The increase rate of breast cancer since 1990 was $19 \%$. While breast cancer for males has a stable rate, its increase rate for females is $24 \%$. In the UK, breast cancer death was 11,400 cases in 2014 , with 75 cases males and the rest were females. breast cancer was the second most common cause of cancer death for women with over 11,300 deaths in 2014. It is also estimated that between 2014 and 2035 the number of breast cancer cases will increase by $2 \%$ in this country (Cancer Research UK, 2014a).

In Europe, studies showed after a peak of cancer death rate in the late 1980, despite differences in different countries, there had been a $10 \%$ decline in mortality rate until 2000 (Karim-Kos et al., 2008; La Vecchia et al., 2009; Bosetti et al.; 2013). However, in 2002, statistics showed that $26 \%$ of global cancer cases belonged to Europe (Parki et al., 2005). In 2006, there were 3,191,600 new cancer cases among them 429,900 were breast cancer cases (about 13.5\%). Among 1,703,000 cancer death, breast cancer was the third cause of death after lung and colorectal cancer with 131,900 (about $7.5 \%$ ) of cancer death cases (Ferlay et al., 2007).

In 2012, data from 54 African countries including three quarters of 47 Sub-Saharan African countries, showed 846,961 (about 6\%) of global new cancer cases, of which 362,037 male and 484,924 female. There were also 591,169 (about 7.2\%) of global cancer deaths, of which 277,849 were male and 313,320 female. The results of this study showed the breast cancer was the leading cancer type for women with a large difference with 133,900 (about $27.6 \%$ ) of all cancers. The study suggested that by 2030 there would be $70 \%$ increase in new cancer cases as well as the cancer death rate in Africa due to population growth and aging (Parkin et al., 2014).

The reports from study in china revealed that cancer was the second most common cause of death, (1 out of 5 deaths with annual 2.82 million new cancer cases and 1.96 million death(Goss et al., 2014). National Cancer Registry Programmed in India monitored six populated regions (Bangalore, Barshi, Bhopal, Chennai, Delhi, and Mumbai) between 1990 and 1996. Their data showed 160,017 new cases of which 79,954 were male and 80,063 female. Their findings indicated that cervical and breast cancers were accountable for $40 \%$ of all cancer cases in women (Nanda, 2001). A more recent study in 2012 by GOLOBOCAN showed annual 1 million new cases of cancer followed by about 700,000 cancer death in India. Since 2009 breast cancer became the most common type of cancer for women and the first cause of cancer death. Breast cancer was the cause of fifth of all female cancer death with 144,937 new cases and 70,218 deaths in 2012 pushing cervical cancer to the second place (Mallath et 
al., 2014).

Another study in 2009 gathered data for 10 Arab countries in the Middle East and North Africa (Salim et al., 2009). Age-standardized breast cancer cases was 38.0 in Jordan, 38.5 in Palestine Authority (Freedman et al., 2006), 11.8 in Saudi Arabia (Bazarbashi et al., 2004), 42.5 in Egypt, 18.8 in Algeria, 29.8 in Tunisia, 14.6 in Oman, 46.8 in Bahrain, 43.1 in Kuwait (Curado et al., 2007), 30.1 in Qatar (Bener et al., 2008). In addition, age-standardized cancer cases 112 in Jordan, 134 in Palestine Authority (Freedman et al., 2006), 58 in Saudi Arabia (Bazarbashi et al., 2004), 122 in Egypt, 85 in Algeria, 106 in Tunisia, 91 in Oman, 143 in Bahrain, 129 in Kuwait (Curado et al., 2007), 87 in Qatar (Bener et al., 2008).

During 1965 to 2000, breast cancer had become the most common type of cancer among women (Mousavi et al., 2007). The cancer registry reports of Iran Ministry of Health for years 2000 to 2006 showed that breast cancer was the most common type of cancer among women with an increase rate of $21.4 \%$ to $24.4 \%$ of all new cancer cases. This report also revealed that breast cancer was the fifth cause of cancer death among Iranian females (Lamyian et al., 2007; Mousavi et al., 2008; Jafari-Koshki et al., 2014). In 2004 another study showed that the mean age of Iranian breast cancer patients about 10 years less than developed countries (Harirchi et al.; 2004).

Overall our findings showed a substantial increase in breast cancer mortality rate in the world during the past 25 years. This could be because of increase in number of new incidents of this type of cancer. This increasing trend is an alarming sign for health policy makers in all countries, in particular in developing and low-income regions which have experienced sharp slopes of increasing breast cancer mortality rate.

\section{References}

American Cancer Society (2017), How common is breast cancer?. Accessed: 01/Jan/2018. URL: https://www.cancer. org/cancer/Breast-cancer/about/how-common-is-Breastcancer.html.

Bazarbashi S, DeVol E, Young S, Al-Eid H, Arteh S (2004), Cancer incidence report Saudi Arabia 1999-2000, National Cancer Registry.

Bener A, Ayub H, Kakil R, Ibrahim W (2008), Patterns of cancer incidence among the population of Qatar: a worldwide comparative study. Asian Pac J Cancer Prev, 9, 19-24.

Bosetti C, Bertuccio P, Malvezzi M, et al (2013), Cancer mortality in Europe, 2005-2009, and an overview of trends since 1980. Ann Oncol, 24, 2657-71.

Cancer Research UK (2014a). Breast cancer statistics-Breast Cancer mortality. Accessed: 01/Jan/2018. URL:http://www. cancerresearchuk.org/health-professional/cancer-statistics/ statistics-by-cancer-type/Breast-cancer.

Cancer Research UK (2014b). Cancer incidence statistics-cancer incidence for all cancers combined. Accessed: 01/Jan/2018.

URL:http://www.cancerresearchuk.org/health-professional/ cancer-statistics/incidenceheading-Zero.

Curado MP, Edwards B, Shin HR, et al (2007). Cancer incidence in five continents, Volume IX., IARC Press, International Agency for Research on Cancer.

Ferlay J, Autier P, Boniol M, et al (2007). Estimates of the cancer incidence and mortality in Europe in 2006. Ann Oncol, 18,
581-92.

Ferlay J, Steliarova-Foucher E, Lortet-Tieulent J, et al (2013), Cancer incidence and mortality patterns in Europe: estimates for 40 countries in 2012. Eur J Cancer, 49, 1374-1403.

Freedman LS, Edwards BK, Ries LA, et al (2006). Cancer incidence in four member countries (Cyprus, Egypt, Israel, and Jordan) of the middle east cancer consortium (mecc) compared with us seer., Cancer incidence in four member countries (Cyprus, Egypt, Israel, and Jordan) of the Middle East Cancer Consortium (MECC) compared with US SEER.

Global Burden of Disease Cancer Collaboration (2017). Global, regional, and national cancer incidence, mortality, years of life lost, years lived with disability, and disability-adjusted life-years for 32 cancer groups, 1990 to 2015: A systematic analysis for the global burden of disease study. JAMA Oncol, 3, 524-48.

Goss PE, Strasser-Weippl K, Lee-Bychkovsky BL, et al (2014). Challenges to effective cancer control in China, India, and Russia. Lancet Oncol, 15, 489-538.

Harirchi I, Karbakhsh M, Kashefi A, Momtahen AJ (2004), breast cancer in Iran: results of a multi-center study. Asian Pac J Cancer Prev, 5, 24-7.

Institute for Health Metrics and Evaluation (2018), 'What countries are in each region?' . Accessed: 21/Jan/2019.

Jafari-Koshki T, Schmid VJ, Mahaki B (2014), Trends of breast cancer incidence in iran during 2004-2008: a Bayesian space-time model. Asian Pac J Cancer Prev, 15, 1557-61.

Jung KW, Won YJ, Oh CM, et al (2017). Cancer statistics in Korea: incidence, mortality, survival, and prevalence in 2014. Cancer Res Treat, 49, 292.

Karim-Kos HE, de Vries E, Soerjomataram I, et al (2008). Recent trends of cancer in Europe: a combined approach of incidence, survival and mortality for 17 cancer sites since the 1990s. Eur J Cancer, 44, 1345-89.

La Vecchia C, Bosetti C, Lucchini F, et al (2009). Cancer mortality in Europe, 2000-2004, and an overview of trends since 1975. Ann Oncol, 21, 1323-60.

Lamyian M, Hydarnia A, Ahmadi F, Faghihzadeh S, Aguilar-Vafaie ME (2007). Barriers to and factors facilitating breast cancer screening among Iranian women: a qualitative study. East Mediterr Health J, 13, 1160-9.

Mallath MK, Taylor DG, Badwe RA, et al (2014). The growing burden of cancer in india: epidemiology and social context, Lancet Oncol, 15, 205-12.

Mousavi SM, Gouya MM, Ramazani R, et al (2008). Cancer incidence and mortality in Iran. Ann Oncol, 20, 556-63.

Mousavi SM, Montazeri A, Mohagheghi MA, et al (2007), breast cancer in Iran: an epidemiological review. Breast $J$, 13, 383-91.

Nanda K (2001). Consolidated report of the population based cancer registries, incidence and distribution of cancer, 1990-1996, National Cancer Registry Program, New Delhi: Indian Council of Medical Research.

Parkin DM, Bray F, Ferlay J, Jemal A (2014), Cancer in Africa 2012. Cancer Epidemiol Biomarkers Prev, 23, 953-66.

Parkin DM, Bray F, Ferlay J, Pisani P (2005). Global cancer statistics, 2002. Cancer J Clin, 55, 74-108.

Salim EI, Moore MA, Al-Lawati JA, et al (2009). Cancer epidemiology and control in the arab world-past, present and future. Asian Pac J Cancer Prev, 10, 3-16.

Sharma R (2019). Breast cancer incidence, mortality and mortality-to-incidence ratio (mir) are associated with human development, 1990-2016:evidence from global burden of disease study 2016. Breast Cancer, 12, 1-18.

Wang H, Naghavi M, Allen C, et al (2016). Global, regional, and national life expectancy, all-cause mortality, and cause-specific mortality for 249 causes of death, 1980-2015: 
Nasrindokht Azamjah et al

a systematic analysis for the global burden of disease study 2015. Lancet, 388, 1459-1544.

World Health Organization (2008). The global burden of disease, 2004 update. Accessed: 05/Jan/2018. URL:http:// www.who.int/healthinfo/global_burden_disease/GBD_ report_2004update_full.pdf.

Zeng H, Zheng R, Zhang S, Zou X, Chen W (2014). Female breast cancer statistics of 2010 in china: estimates based on data from 145 population- based cancer registries. J Thorac Dis, 6, 466.

\section{0ब}

This work is licensed under a Creative Commons AttributionNon Commercial 4.0 International License. 